\title{
Probiotics in irritable bowel syndrome - is the quest for the right strain over? Rapid review of existing guidelines and recommendations
}

\author{
Wojciech Marlicz' ${ }^{1}$, Karolina Skonieczna-Żydecka², Patrycja Krynicka ${ }^{1}$, Igor Łoniewski², Grażyna Rydzewska ${ }^{3,4}$ \\ ${ }^{1}$ Department of Gastroenterology, Pomeranian Medical University, Szczecin, Poland \\ ${ }^{2}$ Department of Biochemical Sciences, Pomeranian Medical University, Szczecin, Poland \\ ${ }^{3}$ Clinical Department of Internal Medicine and Gastroenterology with Inflammatory Bowel Disease Unit, Central Clinical \\ Hospital of the Ministry of the Inferior and Administration, Warsaw, Poland \\ ${ }^{4}$ Collegium Medicum, Jan Kochanowski University, Kielce, Poland
}

Gastroenterology Rev 2021; 16 (4): 369-382

DOI: https://doi.org/10.5114/pg.2021.111766

Key words: probiotics, irritable bowel syndrome, IBS, guidelines.

Address for correspondence: Wojciech Marlicz MD, PhD, FACG, FRCPE, Department of Gastroenterology, Pomeranian Medical University, Szczecin, Poland; e-mail: marlicz@hotmail.com

\begin{abstract}
Irritable bowel syndrome (IBS) - functional gastrointestinal disorder (FGIDs) and disorder of gut-brain interaction (DGBIs) - has emerged as an important medical problem with an impact on health care systems, affecting patients' quality of life. The management of IBS consists of pharmacological and non-pharmacological treatments; however, the data of their long-term efficacy are scarce. Modulation of gastrointestinal microbiota, by means of probiotics and prebiotics, is often sought and advertised as a popular treatment modality in IBS. Faecal microbiota transplantation (FMT) awaits recommendations for IBS treatment and requires more methodological assessments. To date, numerous guidelines and recommendations have been published on the role of probiotics in IBS. Because no probiotic claim for probiotics in foods has yet been granted by the European Food and Safety Authority (EFSA), medical practitioners still recommend probiotics on the basis of available literature and recommendations released by independent health authorities. We aimed to summarize published formal recommendations and guidelines regarding the clinical effectiveness of available probiotic strains and conduct a random-effects meta-analysis of outcomes for which $\geq 2$ studies contributed data on the same probiotic strain recommended to adults with IBS. Based on available and most recent guidelines, we report that probiotics, as a group, may be an effective treatment for global symptoms and abdominal pain in IBS, with the strongest effect for genus Lactobacillus. Our current and updated meta-analysis is in line with several reports documenting significant effects of Lactobacillus plantarum (Lp299v) in reducing the risk of global symptoms and their persistence, which could assist clinicians in making the choice for the right probiotic strain in IBS patients.
\end{abstract}

\section{Introduction}

Irritable bowel syndrome (IBS) has emerged as an important medical problem with a major impact on global health care systems and patients' quality of life. IBS lies in the spectrum of functional gastrointestinal disorders (FGIDs), recently named disorders of gutbrain interaction (DGBIs). According to Rome IV criteria, FGIDs/DGBIs are classified by gastrointestinal symptoms related to a combination of any of the following: i) motility disturbances, ii) visceral hypersensitivity, iii) altered mucosal and immune function, iv) gut microbiome, and/or v) central nervous system functioning [1, 2].
For the 2 most common DGBIs syndromes - IBS and functional dyspepsia (FD) - the worldwide prevalence ranges from $1.1 \%$ to $57 \%$, which could reflect the scale of methodological heterogeneity, differences among surveyed populations, or methodological biases among reported studies [3]. Several factors could contribute to various biases of outcomes in IBS studies, including (yes or no) reporting of the following: i) comorbid conditions, ii) dietary habits, iii) mental health status, iv) ethnicity, v) timing of enrolment, vi) recording of menses, vii) the use of a run-in periods, viii) effect size and formal statistical power analysis, ix) clinical endpoints, $x$ ) fluctuations in symptoms, and $x i$ ) the potential for 
wide variations in bowel habits [4]. In the most recent large-scale multinational study, comprising 33 countries, the investigators reported that more than $40 \%$ of persons worldwide suffer from FGIDs/DGBIs with IBS rates between $3 \%$ and $5 \%$ in most countries [3].

The treatment of IBS consists of various pharmacological and non-pharmacological modalities; however, the data of long-term follow-up for most of them are scarce $[5,6]$. Many patients initially benefit from placebo effect $[7,8]$ but respond poorly to longer-term treatments [9], either do not tolerate or not respond to pharmacotherapy, and may be worried by potential adverse events [10]. Finally, some patients may become dissatisfied with doctors' advice and treatment options. Modulation of gastrointestinal microbiota by means of probiotics and prebiotics is often sought, advertised as a popular treatment modality among IBS patients [11]. Faecal microbiota transplantation (FMT) currently is not routinely recommended for IBS treatment and requires further methodological assessments [12].

To date, experts, authorities, and medical societies conduct and aim to publish well-powered, appropriately-designed studies and offer their interpretation of data with a high level of evidence (i.e. systematic reviews and meta-analyses) to draw conclusions or develop guidelines and recommendations. Until now numerous guidelines and recommendations have been published concerning the role of probiotics in IBS. Of note, claims for probiotics in foods in the European Union (EU) are yet to be granted by the European Food and Safety Authority (EFSA). Therefore, medical experts still recommend probiotics on the basis of scientific literature and recommendations preferably released by independent and non-commercial health authorities.

In the last decade numerous guidelines and recommendations evaluating the utility of probiotics in IBS have been published [13-16]. However, not all reports available on the Internet are adhering to standards of evidence-based medicine (EBM), lacking scientific validity, and presenting contrasting conclusions, which makes clinical judgment difficult. Sadly, uncertainty among medical professionals regarding the choice of probiotics in IBS still exists. Similarly, patients' queries to medical practitioners regarding the role of probiotics in their digestive misalignments, including IBS symptoms, are frequent. The questions raised are often difficult to answer because most of the probiotics available on the market are registered as food supplements, frequently with no clear evidence of efficacy.

\section{Aim}

Therefore, we aimed to analyse published recommendations and guidelines regarding the clinical effec- tiveness of various probiotic strains recommended to adults with IBS.

\section{Material and methods}

The strategy to evaluate the papers relevant to our aims comprised the following steps: 1) searching for relevant recommendations/guidelines, 2) applying exclusion criteria, and 3) synthesizing guideline recommendations to identify issues potentially relevant to the efficacy of probiotics in IBS.

\section{Data Sources and Search Terms}

For the present rapid review, we conducted a search in PubMed/Embase/Google Scholar up to 15 May 2021, using the following search terms:

Embase: ('irritable bowel syndrome' OR ibs OR 'digestive system function disorder'/exp OR 'digestive system function disorder' OR 'functional gastrointestinal disorder' OR 'gastrointestinal dysfunction' OR 'gastrointestinal function disorder' OR 'gastrointestinal tract function disorder') AND 'treatment' OR 'management' OR 'drugs' OR 'agents' OR 'therapy' OR ‘clinical practice' OR 'probiotic agent'/exp OR 'probiotic' OR 'probiotic agent' OR 'probiotics' OR 'synbiotic agent'/exp OR 'synbiotic' OR 'synbiotic agent' OR 'synbiotics' OR 'microbes' OR 'beneficial microbes' OR 'beneficial bacteria') AND ('systematic review'/exp OR 'review, systematic' OR 'systematic review' OR 'practice guideline'/exp OR 'recommendations'/exp).

PubMed: (irritable bowel syndrome OR ibs OR digestive system function disorder OR digestive system function disorder OR functional gastrointestinal disorder OR gastrointestinal dysfunction OR gastrointestinal function disorder OR gastrointestinal tract functiondisorder) AND (treatment OR management OR drugs OR agents OR therapy OR clinical practice OR probiotic OR probiotic agent ORprobiotics OR synbiotic agent OR synbiotic OR synbiotic agent OR synbiotics OR microbes OR beneficial microbes OR beneficial bacteria) AND (systematic review OR review, systematic OR systematic review OR practice guideline OR recommendations).

The electronic search was supplemented by a manual review of the reference lists from eligible publications and relevant reviews. Taking into account recently published guidelines exploring the role of probiotics in IBS by major gastrointestinal societies, the most recent being from the American Gastroenterology Association (AGA) $[17,18]$ and the British Society of Gastroenterology (BSG) [19], we decided to present to outcomes of our analysis in the form of a rapid review and synthesis of data, in which parts of the systematic review process are either simplified or omitted to obtain the information in a timely manner. Because our study is 
a rapid review the results are potentially not exhaustive. The eligible papers were chosen by 2 authors (PK and WM).

\section{Inclusion and exclusion criteria}

Our inclusion criteria were as follows:

1) documents (recommendations/guidelines) in which the effectiveness of probiotics in patients with IBS was analysed;

2) documents (recommendations/guidelines) in adult humans (> 18 years old);

3) documents in English, German, and Polish;

4) recommendations/recommendations of scientific societies, medical organizations, and expert groups that are formalized.

Exclusion criteria were:

1) reports with no IBS-related outcome;

2) reports with no probiotics-related outcome;

3) commercial, advertisement, etc. recommendations.

\section{Outcomes}

After selecting the papers, we abstracted data on the following sections: i. general statement of the authorities, ii. level of recommendation along with the quality of evidence, and iii. recommended probiotic strains. The Excel files with abstracted data completed by each investigator were compared with the content of the others' files, and any discrepancies were solved by the last author by consensus.

\section{Pooling results}

We conducted a random effects [20] meta-analysis of outcomes for which $\geq 2$ studies contributed data on the same probiotic strain, using Comprehensive Meta-Analysis V3 (http://www.meta-analysis.com). We explored study heterogeneity using the $\chi^{2}$ test of homogeneity, with $p<0.05$ indicating significant heterogeneity. All analyses were 2-tailed with alpha equal to 0.05. Categorical outcomes (global symptoms and abdominal pain persistence) were analysed by calculating the pooled risk ratio (RR). We inspected funnel plots and used Egger's regression test [21] and the Duval and Tweedie's trim and fill method [22] to quantify whether publication bias could have influenced the results.

\section{Results and discussion}

We identified 17 papers presenting guidelines or recommendations for probiotics in irritable bowel syndrome. Overall, in 13 guidelines/recommendations, probiotics were recommended, whilst in 4 statements no recommendation or "advise to use probiotics only in the content of clinical trial" were given. The position state- ments of societies' recommendations regarding the use of probiotic strains in IBS are presented in Table I (synthesis of the recommendations related to probiotic therapy in IBS). The dose and duration of probiotic therapy reported across IBS guidelines are presented in Table II. The quality of evidence among selected guidelines and recommendations varied from very low to moderate. The level of recommendation ranged from no recommendation (AGA 2021) $[17,18]$, through conditional (CAG 2019) [16], to strong recommendation (JSGE 2021) [14]. Most of the reports followed systematic methods to search for evidence. Moreover, a diverse multidisciplinary working group of clinicians and academics with experience in primary, secondary, or tertiary care, as well as dietetics, were involved in some more recent guidelines [17] working with the collaboration of IBS patient networks, using the Grading of Recommendations Assessment, Development, and Evaluation system to evaluate the strength of the recommendations and the overall quality of evidence [17]. However, the majority of positive recommendations for probiotics in IBS declared insufficient evidence for any particular probiotic strains or species.

The most recent guidelines of the British Society of Gastroenterology (BSG) recommended dietary advice as a first-line strategy in all patients with IBS (recommendation: strong, quality of evidence: weak) and probiotics, as a group, as an effective treatment for global symptoms and abdominal pain in IBS (recommendation: weak, quality of evidence: very low) [19].

The study published recently by the American Gastroenterological Association (AGA) presented contrasting guidelines (no recommendations for probiotics in IBS due to insufficient data and knowledge gap) utilizing a process for developing clinical practice guidelines following the Grading of Recommendations Assessment, Development, and Evaluation (GRADE) approach and best practices as outlined by the National Academy of Science (formerly the Institute of Medicine) [17]. In their recent guideline summary the AGA states: "Although there has been a substantial number of studies examining probiotics in various gastrointestinal diseases, the studies have been extremely varied, including differences in the strain of microbes used, dose, and route of administration, as well as the research methodology, including differences in the reporting of end points and outcomes. Furthermore, most of the studies with probiotics involved a relatively small number of patients compared to trials investigating the effects of pharmacological interventions. Conclusions drawn from meta-analyses or systematic reviews can be misleading if different studies with different patient populations, different reported end points and outcomes, or differ- 


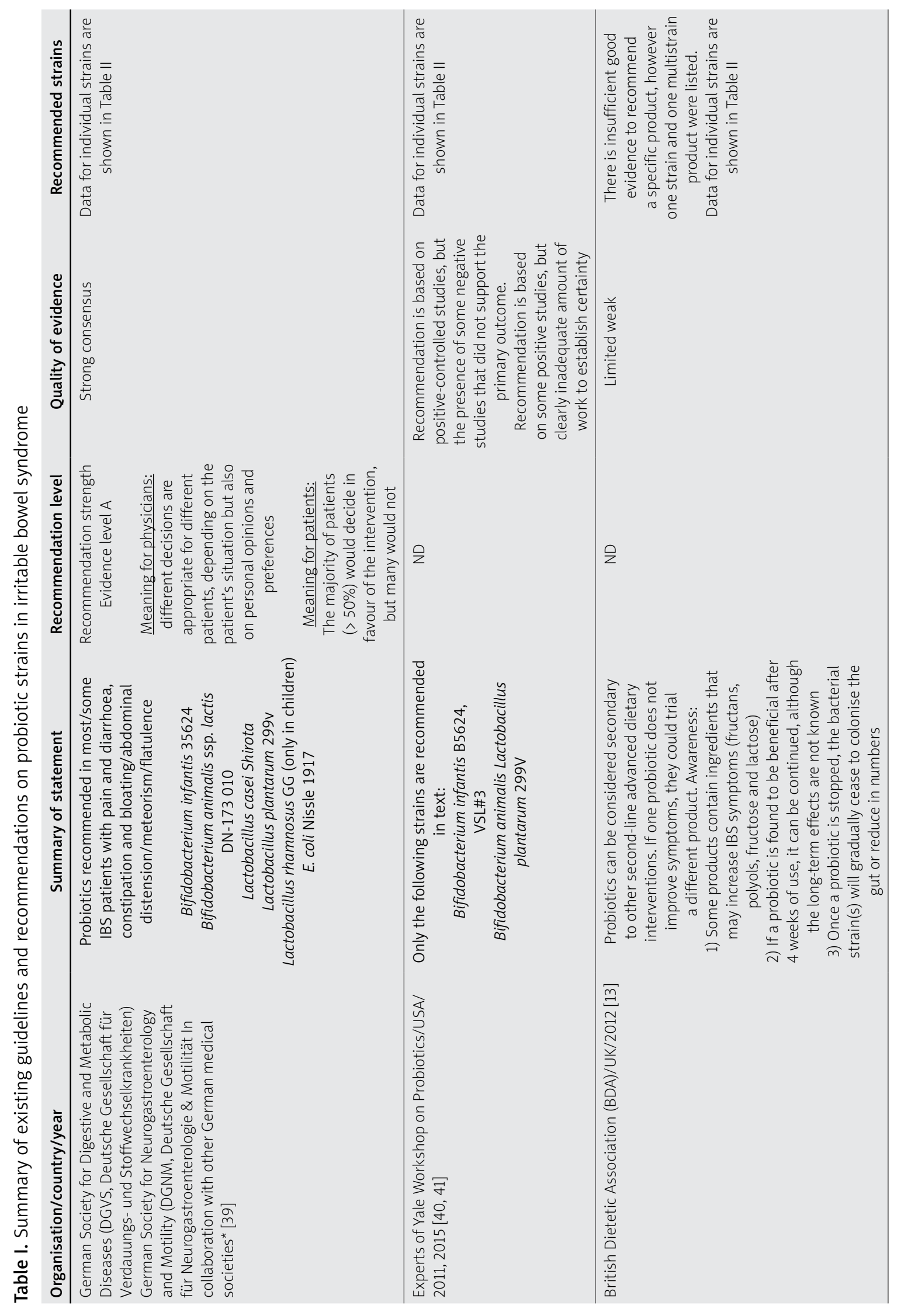




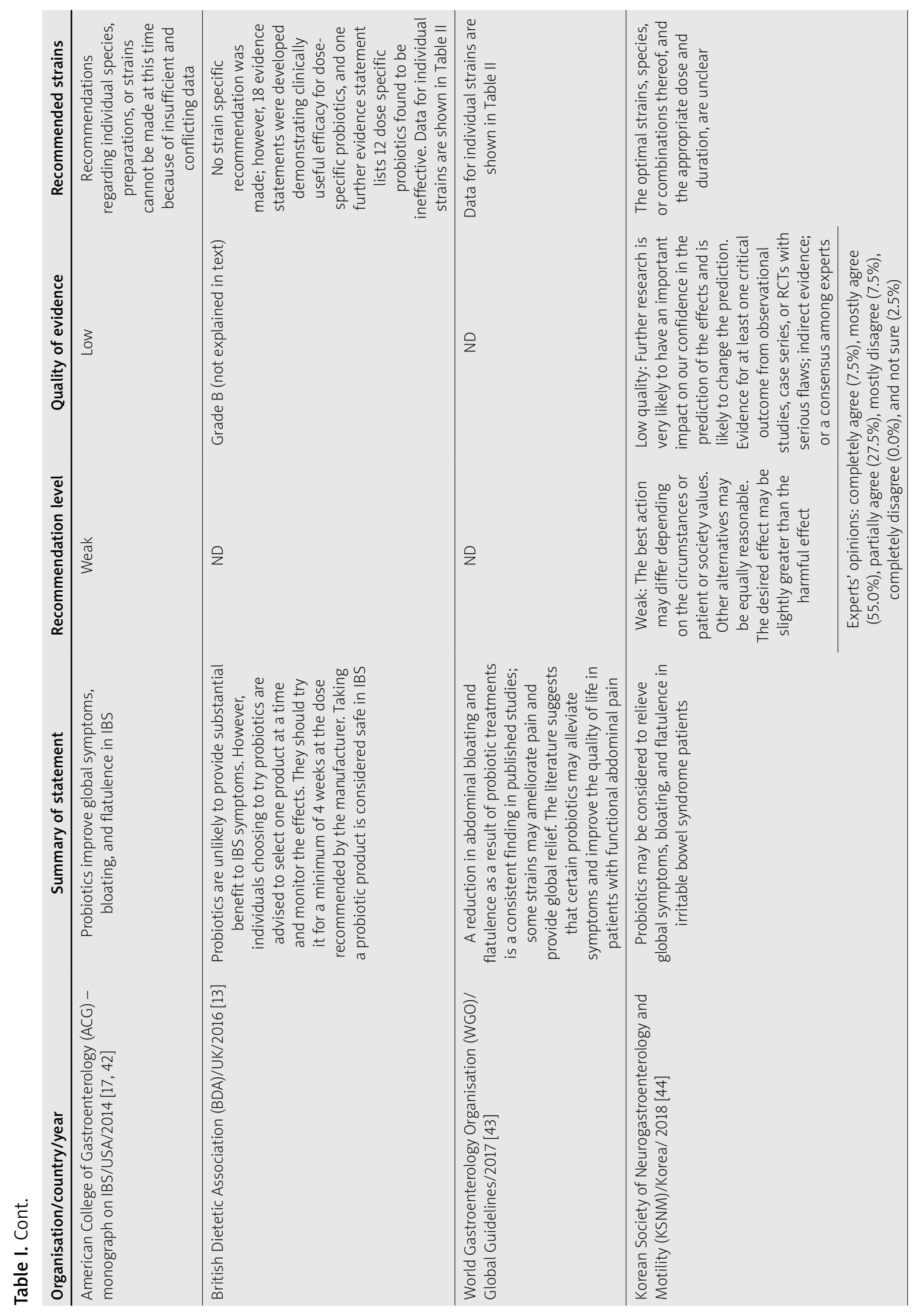




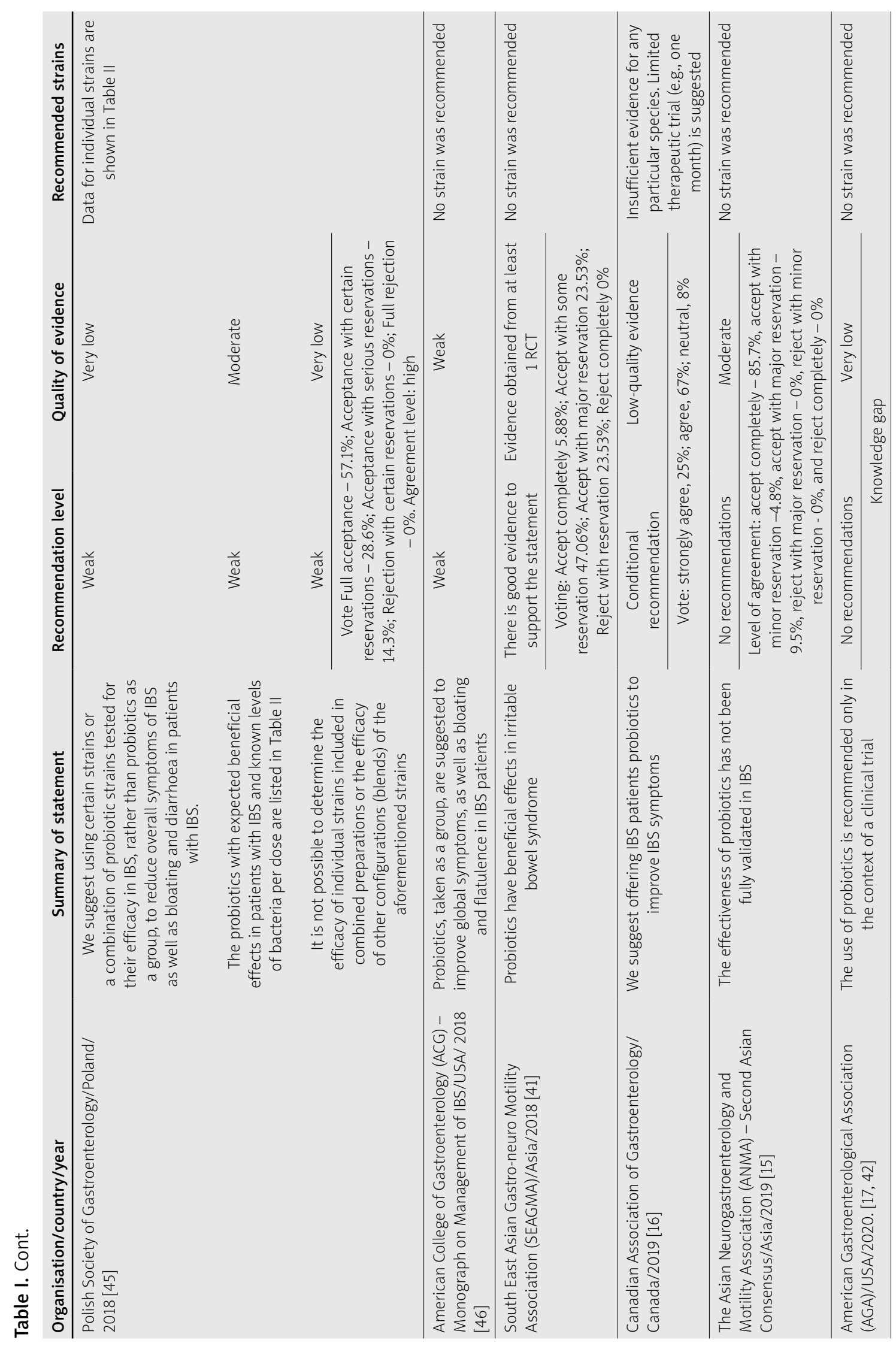




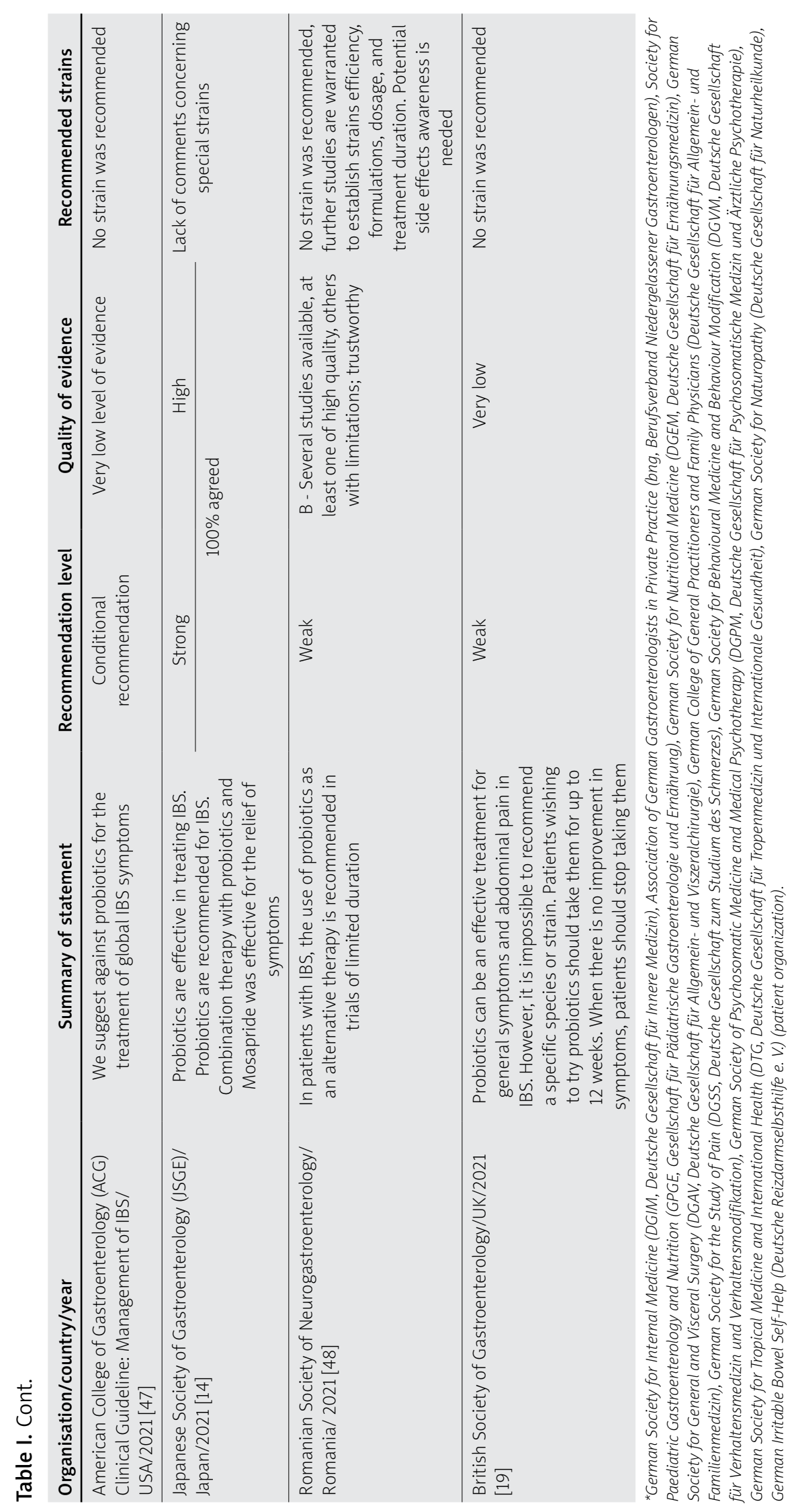




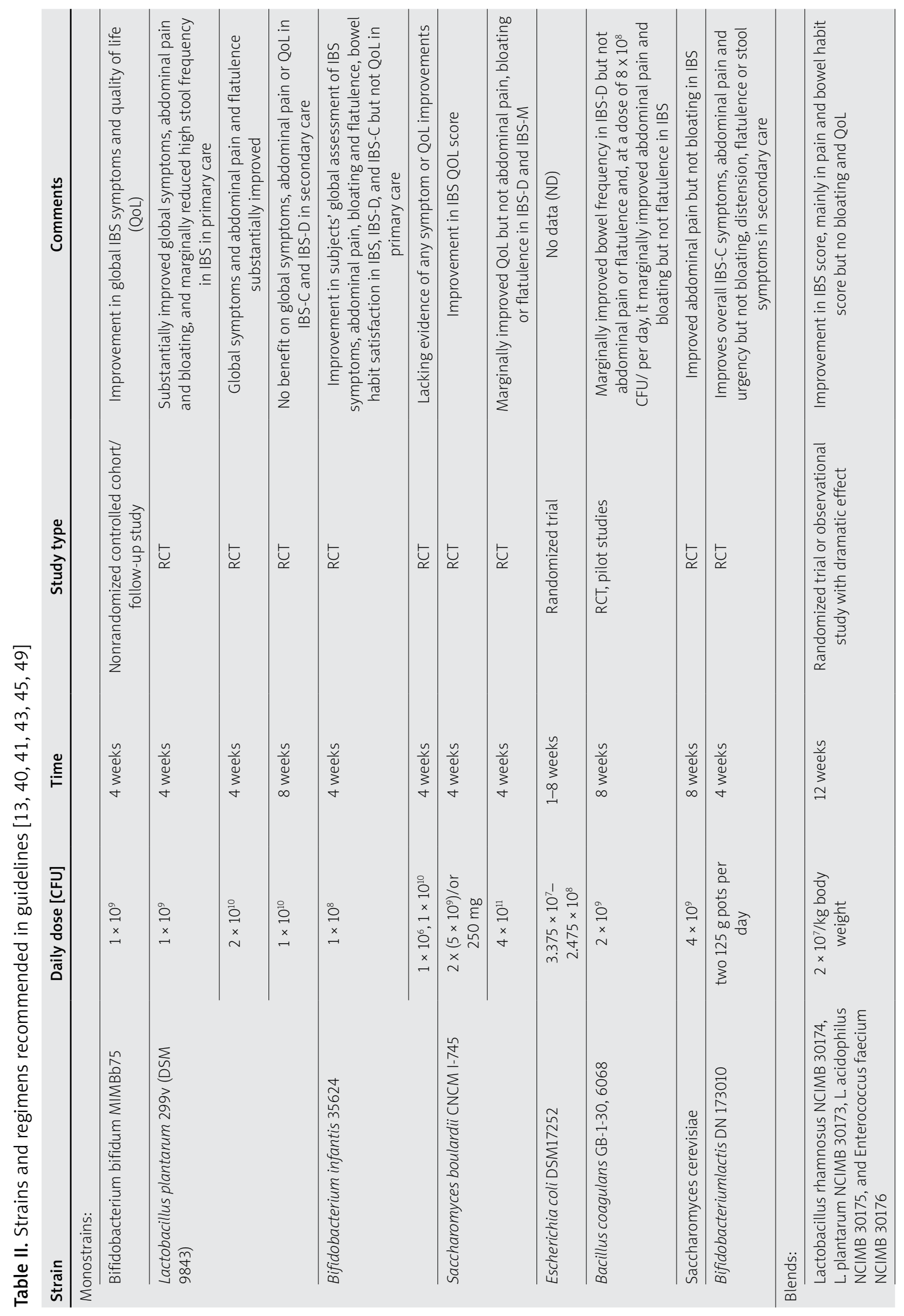




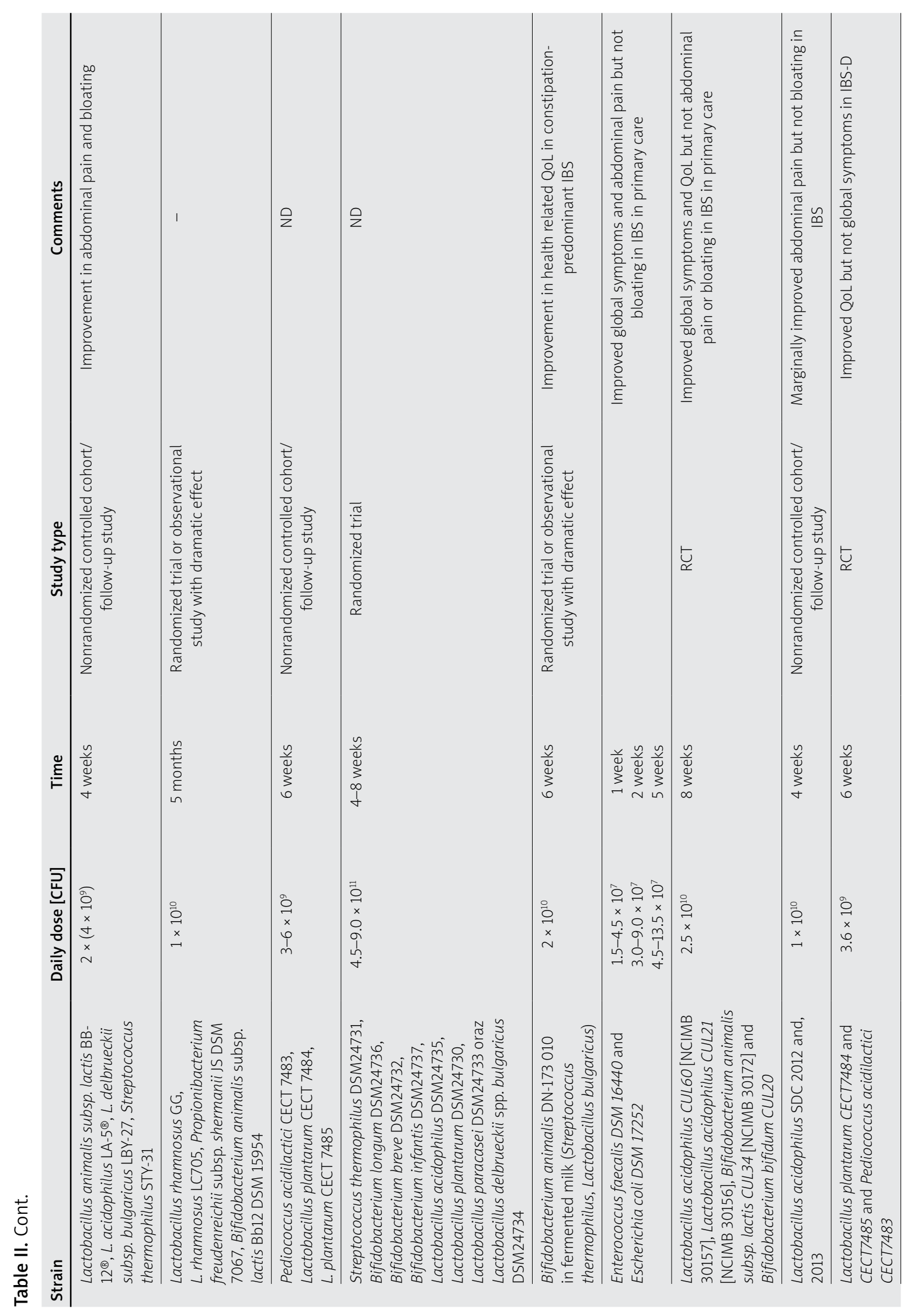




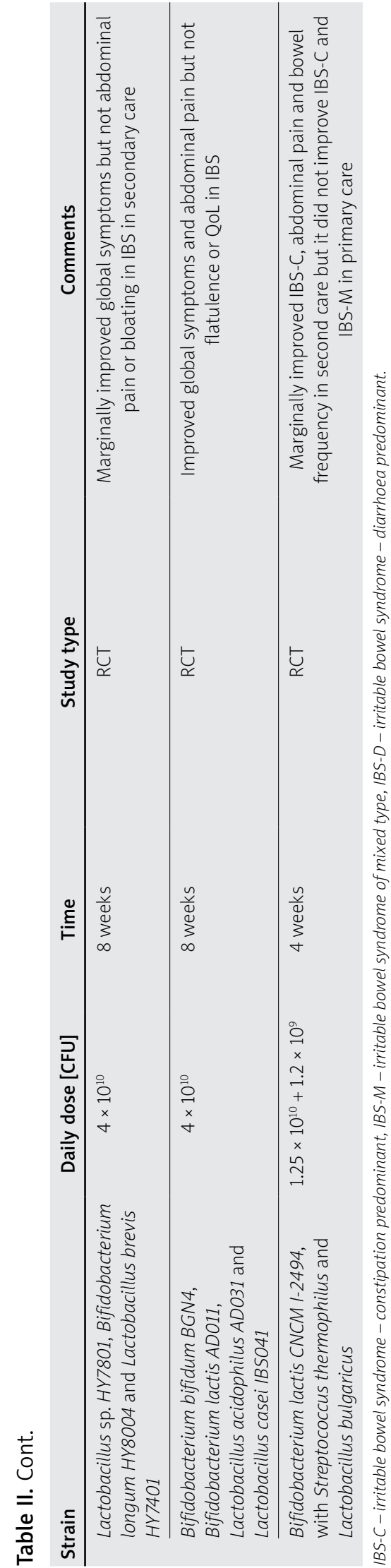

ent strains or combinations of probiotics are grouped together inappropriately" [17].

Of all the analysed statements and recommendations, we paid attention to the most recent BSG guidelines on the management of IBS [19], which followed those recently released by AGA [17]. In contrast to the AGA recommendations, the BSG state that probiotics, as a group, may be an effective treatment for global symptoms and abdominal pain in IBS. Subgroup analyses of 45 randomized clinical trials (RCTs), randomizing 6352 patients according to type of probiotic used, demonstrated the strongest effects on global symptoms or abdominal pain for Lactobacillus (relative risk (RR) $=0.75 ; 95 \%$ confidence interval $(\mathrm{Cl}): 0.60-0.94)$. However, variations in study design, strain, and species of probiotic used, and heterogeneity between studies did not allow for specific strain recommendations. Probiotic effects are strain dependent and should be documented in at least one RCT, preferably accompanied by confirmatory trials [23, 24]. Despite an overall large number of RCTs in IBS, only a few of them used the same probiotic strain. Therefore, we conducted subgroup analysis on Lactobacillus plantarum 299v (Lp299v) in comparison to other Lactobacilli reported in the most recent guidelines [19]. For 11 meta-analysed studies including 843 patients and investigating the effect of Lactobacillus species for global symptoms and abdominal pain in IBS, $82 \%$ heterogeneity $\left(l^{2}\right)$ was reported. Among them, 3 RCTs including 240 patients investigated the effect of Lp299v, reporting RR $=0.67(p=0.04)$ with $R^{2}=62.5$ $(p=0.07)$. For other Lactobacilli, RR $0.79(p=0.08)$ with $R^{2}=80.3(p<0.001)$ were found. Publication bias for all Lactobacilli studies was not relevant $(p=0.59)$. A similarly low and statistically non-relevant $P^{2}(51 \%, p=0.09)$ was reported for IBS studies investigating the effect of Bifidobacteria, with 2 studies using the same probiotic strain Bifidobacterium bifidium HI-MIMBb75, but with overall less risk reduction $(\mathrm{RR}=0.80 ; 95 \% \mathrm{Cl}: 0.70-$ $0.91)$. In the case of Saccharomyces, 3 studies were meta-analysed with very high heterogeneity $\left(I^{2}=96 \%\right)$ and no significant effect on IBS global symptoms or abdominal pain $(p=0.18)$. Our current subgroup analysis (Figures $1 \mathrm{~A}, \mathrm{~B}$ ) is in line with previous meta-analysis [25] reporting significant effects of Lp299v in reducing the risk of symptom persistence. Moreover this meta-analysis shows that a total favourable effect of Lactobacilli in IBS is driven by Lp299v. These observations "should considerably assist clinicians in making recommendations regarding the choice of probiotic that a patient should take" [26]. Of relevance are studies investigating the effects of probiotics in IBS in real-life settings. Of those reported in real life Lp299v significantly alleviated the global IBS symptoms, achieving maximum 


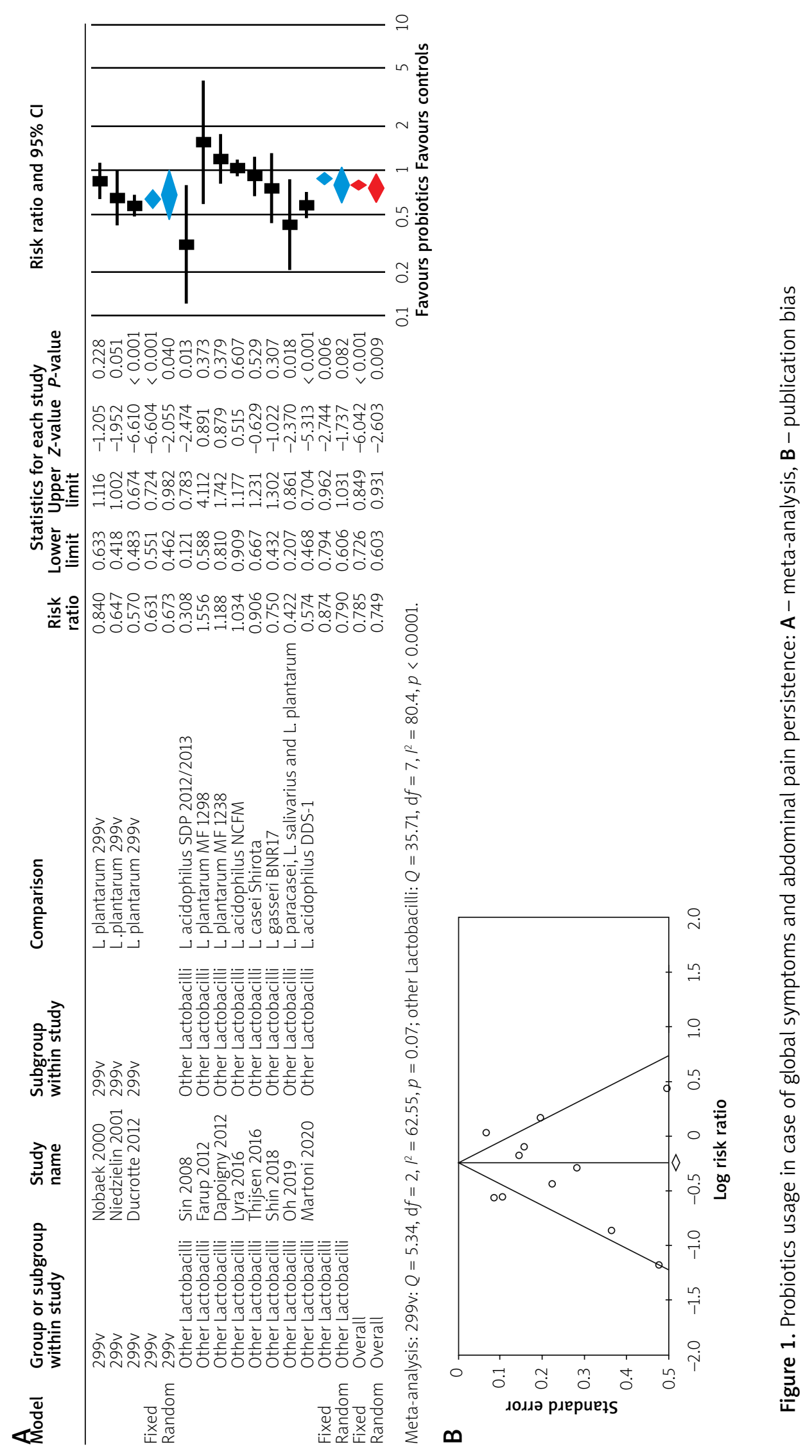




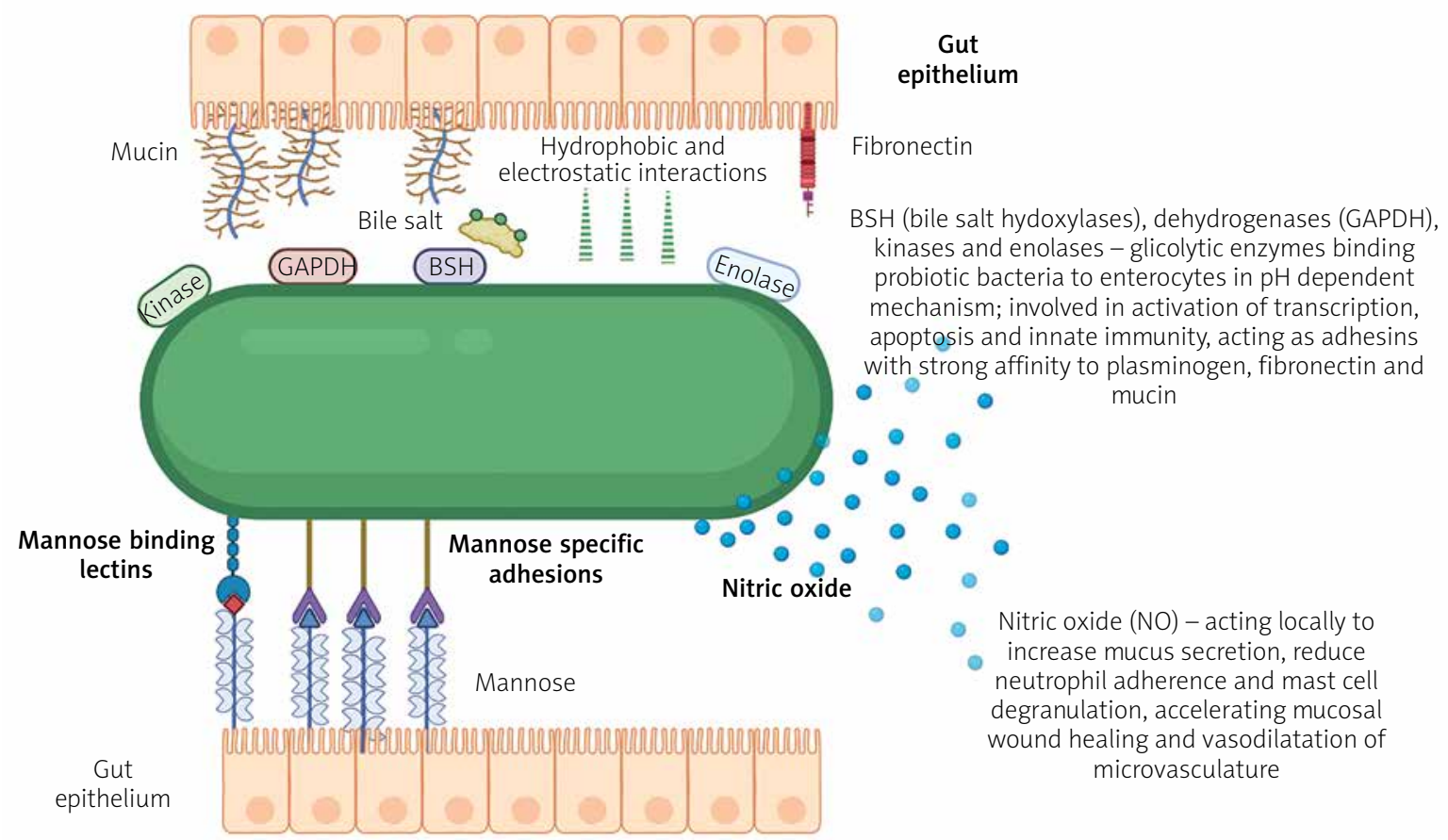

Figure 2. Adhesive properties and mechanism of action of LP299v probiotic strain [28, 30, 31]. Some mechanisms of probiotic action might be widespread; others frequently observed, yet others may be rare and characteristic for only a few strains of a given species. Evidence is mounting. Major probiotic mechanisms of action include enhancement of various elements of the epithelial barrier, increased adhesion to intestinal mucosa, concomitant inhibition of pathogen adhesion, competitive exclusion of pathogenic microorganisms, production of anti-microorganism substances, and modulation of the immune and neural systems. The main mechanisms of action of $S$. boulardii include inhibition of activities of bacterial pathogenic products, trophic effects on the intestinal mucosa, as well as modification of host signalling pathways involved in inflammatory and non-inflammatory intestinal diseases. The mechanism involved in anti-mutagenic activities is the ability of bifidobacteria to bind to the mutagens of microbial cells

effect within 12 weeks of treatment [27], which is in line with the most recent guidelines as for time of probiotics administration [1]. These effects could be facilitated through unique mechanisms already known and described [28-32] for the Lp299v strain (Figure 2). Of importance, $L p 299 \mathrm{~V}$ fulfils the regulatory statements of Binda et al., which are as follows: "Probiotic strains must be (i) sufficiently characterized; (ii) safe for the intended use; (iii) supported by at least one positive human clinical trial conducted according to generally accepted scientific standards or as per recommendations and provisions of local/national authorities when applicable; and (iv) alive in the product at an efficacious dose throughout shelf life" [24]. Although the non-viable $B$. bifidum HI-MIMBb75 strain, also evaluated by Vasant et al. [19] in BSG recommendations, substantially alleviated IBS and its symptoms in real-life, according to the International Scientific Association for Probiotics and Prebiotics (ISAPP), dead microbes should not come under the probiotic classification [8]. As stated in current BSG guidelines [19], adverse event rates were similar in the probiotic and placebo arms, which is in agreement with the Agency for Healthcare Research and Quality (AHRQ), which reported no increased RR of the overall number of experienced adverse events $(\mathrm{RR}=1.00 ; 95 \% \mathrm{Cl}: 0.93-1.07 ; p=0.999)$, gastrointestinal, infections, and other adverse events, including serious adverse events ( $R R=1.06 ; 95 \% \mathrm{Cl}: 0.97-1.16 ; p=$ 0.201 ), associated with probiotic intake [33]. This is important because consumer awareness on probiotics is rising, as is their intake for general digestive or health reasons [34]. Therefore, as well as guidelines, quality manufacturing control is critical for probiotic safety and efficacy. According to the FAO/WHO, probiotics are "live microorganisms which when administered in adequate amounts confer a health benefit on the host" [35]. All constituents of this definition should be taken into consideration when defining probiotic manufacturing and 
quality requirements. Most probiotics available on the market contain Lactobacilli or Bifidobacteria, as well as few other types of microorganisms. The choice of probiotic strains in the final probiotic product is based on biological, technological, and legislative factors. Probiotics undergo several technological and industrial steps, which include lyophilization (freeze-drying), with the potential to seriously alter their viability and end-product stability. Once ingested, probiotics initiate numerous physiological effects in the gastrointestinal tract (e.g. biofilm formation, release of bioactive substances, competing with other microorganisms). The evaluation of an effective probiotic dose is experimental. According to available literature the daily dose of ingested probiotic bacteria should range between $10^{8}$ and $10^{11} \mathrm{CFU} /$ day live bacteria [36]. The declared number of viable bacteria in the product should be maintained through the shelf-life date. Because the probiotic-related health properties are strain and dose dependent, claims should be documented in well-planned and -conducted, randomized, placebo-controlled clinical trials [37]. Moreover, translucent legislative procedures are needed for probiotics, currently classed in the nomenclature either as food or drugs.

\section{Conclusions}

Probiotics, as a group, may be an effective treatment for global symptoms and abdominal pain in patients with IBS. Subgroup analyses of available randomized clinical trials (RCTs), according to the type of probiotic used, demonstrated the strongest effects on global symptoms or abdominal pain in patients with IBS for the genus Lactobacillus, but this effect is driven by strain Lp299v. Lp299v significantly alleviates the global IBS symptoms, achieving maximum effect within 12 weeks of treatment, which is in line with the most recent and major gastrointestinal society guidelines as for the time of probiotics administration. Our current analysis is in line with previous reports documenting significant effects of Lp299v in reducing the risk of global symptoms and their persistence, which could assist clinicians in making the correct choice of probiotic strain in IBS patients [38]. Head-to-head studies evaluating the efficacy of the same or different probiotic strains in IBS patients are lacking, which stand behind limitations of various meta-analyses. Probiotics have good safety profile but require restrictive manufacturing procedures and high quality control standards.

\section{Conflict of interest}

Wojciech Marlicz and Igor Łoniewski are cofounders and shareholders in Sanprobi - manufacturer of probiotics. Karolina Skonieczna-Żydecka received remuner- ations for speaking and research engagements from Sanprobi. The content of this study was neither influenced nor constrained by these facts. The other authors declare no conflict of interest.

\section{References}

1. Drossman DA, Hasler WL. Rome IV - functional GI disorders: disorders of gut-brain interaction. Gastroenterology 2016; 150: 1257-61.

2. Kaminski M, Skonieczna-Zydecka K, Loniewski I, et al. Are probiotics useful in the treatment of chronic idiopathic constipation in adults? a review of existing systematic reviews, meta-analyses, and recommendations. Gastroenterology Rev 2020; 15: 103-18.

3. Sperber AD, Bangdiwala SI, Drossman DA, et al. Worldwide prevalence and burden of functional gastrointestinal disorders: results of Rome Foundation Global Study. Gastroenterology 2021; 160: 99-114.e3

4. Miller LE. Study design considerations for irritable bowel syndrome clinical trials. Ann Gastroenterol 2014; 27: 338-45.

5. Cui J, Lin Z, Tian H, et al. Long-Term follow-up results of fecal microbiota transplantation for irritable bowel syndrome: a single-center, retrospective study. Front Med 2021; 8: 710452.

6. Weerts ZZRM, Vork L, Mujagic Z, et al. Reduction in IBS symptom severity is not paralleled by improvement in quality of life in patients with irritable bowel syndrome. Neurogastroenterol Motil 2019; 31: e13629.

7. Charlesworth JEG, Petkovic G, Kelley JM, et al. et al. Effects of placebos without deception compared with no treatment: a systematic review and meta-analysis. J Evid Based Med 2017; 10: 97-107.

8. Kaptchuk TJ, Friedlander E, Kelley JM, et al. et al. Placebos without deception: a randomized controlledtrial in irritable bowel syndrome. PLoS One 2010; 5: e15591.

9. Putu D, Shoveller J, Montaner J, et al. Evaluating when and why patients discontinue chronic therapy for irritable bowel syndrome with constipation and chronic idiopathic constipation. Physiol Behav 2016; 176: 139-48.

10. Joć E, Mądro A, Celiński K, et al. Quality of life of patients with irritable bowel syndrome before and after education. Psych Pol 2015; 49: 821-33.

11. Gendi R, Jahan N. Pharmacological and non-pharmacological treatments of irritable bowel syndrome and their impact on the quality of life: a literature review. Cureus 2020; 12: e9324.

12. El-Salhy M, Hausken T, Hatlebakk JG. Current status of fecal microbiota transplantation for irritable bowel syndrome. Neurogastroenterol Motil 2021, doi:10.1111/nmo.14157.

13. McKenzie YA, Bowyer RK, Leach $\mathrm{H}$, et al. British Dietetic Association. Systematic review and evidence-based practice guidelines for the dietary management of irritable bowel syndrome in adults (2016 update). J Human Nutrition Dietetics 2016; 29: 549-75.

14. Fukudo S, Okumura T, Inamori M, et al. Evidence-based clinical practice guidelines for irritable bowel syndrome 2020. J Gastroenterol 2021; 56: 193-217.

15. Gwee KA, Gonlachanvit S, Ghoshal UC, et al. Second Asian Consensus on irritable bowel syndrome. J Neurogastroenterol Motil 2019; 25: 343-62. 
16. Moayyedi P, Andrews CN, MacQueen G, et al. Canadian Association of Gastroenterology Clinical Practice Guideline for the Management of Irritable Bowel Syndrome (IBS). J Canad Assoc Gastroenterol 2019; 2: 6-29.

17. Su GL, Ko CW, Bercik P, et al. AGA Clinical Practice Guidelines on the role of probiotics in the management of gastrointestinal disorders. Gastroenterology 2020; 159: 697-705.

18. Bruffaerts R, Mortier P, Kiekens G, et al. AGA technical review. Physiol Behav 2017; 176: 139-48.

19. Vasant DH, Paine PA, Black CJ, et al. British Society of Gastroenterology Guidelines on the management of irritable bowe syndrome. Gut 2021; 70: 1214-40.

20. DerSimonian R, Laird N. Meta-analysis in clinical trials. Control Clin Trials 1986; 7: 177-88.

21. Egger M, Smith GD, Schneider M, Minder C. Bias in meta-analysis detected by a simple, graphical test. Br Med J 1997; 315 629-34.

22. Shi L, Lin L, Omboni S. The trim-and-fill method for publication bias: practical guidelines and recommendations based on a large database of meta-analyses. Medicine 2019; 98 e15987.

23. de Simone C. The unregulated probiotic market. Clin Gastroenterol Hepatol 2019; 17: 809-17.

24. Binda S, Hill C, Johansen E, et al. Criteria to qualify microorganisms as "probiotic" in foods and dietary supplements. Front Microbiol 2020; 11: 1662.

25. Ford AC, Quigley EMM, Lacy BE, et al. Efficacy of prebiotics, probiotics, and synbiotics in irritable bowel syndrome and chronic idiopathic constipation: systematic review and meta-analysis. Am J Gastroenterol 2014; 109: 1547-62.

26. Whelan K. Editorial: the importance of systematic reviews and meta-analyses of probiotics and prebiotics. Am J Gastroentero 2014; 109: 1563-5.

27. Weersma RK, Zhernakova A, Fu J. Treatment of IBS Lactobacillus. Gut 2020; 69: 1510-9.

28. Marlicz W, Yung DE, Skonieczna-Żydecka K, et al. From clinical uncertainties to precision medicine: the emerging role of the gut barrier and microbiome in small bowel functional diseases. Expert Rev Gastroenterol Hepatol 2017; 11: 961-78.

29. Larsson SC, Håkansson N, Wolk A. Healthy dietary patterns and incidence of biliary tract and gallbladder cancer in a prospective study of women and men. Eur J Cancer 2017; 70: 42-7.

30. Nordström EA, Teixeira C, Montelius C, et al. Lactiplantibacillus Plantarum 299v (LP299V®): three decades of research. Benef Microbes 2021; 12; 441-65.

31. Holst B, Glenting J, Holmstrøm K, et al. Molecular switch controlling expression of the mannose-specific adhesin, Msa, in Lactobacillus plantarum. Appl Environ Microbiol 2019; 85 : e02954-18.

32. Holst B, Glenting J, Holmstrøm K, et al. Molecular switch controlling expression of the mannose-specific adhesin, Msa, in Lactobacillus plantarum. Appl Environ Microbiol 2019; 85 e02954-18.

33. Hempel S, Newberry S, Ruelaz A, et al. Safety of probiotics used to reduce risk and prevent or treat disease. Evid Rep Technol Assess 2011; 200: 1-645.
34. O’Connor LE, Gahche JJ, Herrick KA, et al. Nonfood prebiotic, probiotic, and synbiotic use has increased in US adults and children from 1999 to 2018. Gastroenterology 2021; 161: 47686.e3.

35. Hill C, Guarner F, Reid G, et al. Expert Consensus Document: The International Scientific Association for Probiotics and Prebiotics Consensus Statement on the scope and appropriate use of the term probiotic. Nature Rev Gastroenterol Hepatol 2014; 11: 506-14.

36. Marco ML, Hill C, Hutkins R, et al. Should there be a recommended daily intake of microbes? J Nutrition 2020; 150: 3061-7.

37. Fenster K, Freeburg B, Hollard C, et al. The production and delivery of probiotics: a review of a practical approach. Microorganisms 2019; 7: 83

38. McFarland LV, Karakan T, Karatas A. Strain-specific and outcome-specific efficacy of probiotics for the treatment of irritable bowel syndrome: a systematic review and meta-analysis. EClinicalMedicine 2021; 41: 101154.

39. Layer P, Andresen V, Pehl C, Allescher H. Irritable Bowel Syndrome: German Consensus Guidelines on definition, pathophysiology and management. Z Gastroenterol 2011; 49: 237-93.

40. Floch $\mathrm{MH}$, Walker WA, Madsen K, et al. Recommendations for probiotic use - 2011 update. J Clin Gastroenterol 2011; 45: 168-71.

41. Floch $M H$, Walker WA, Sanders E, et al. Recommendations for probiotic use - 2015 update: proceedings and consensus opinion: erratum. J Clin Gastroenterol 2016; 50: 800.

42. Gwee KA, Lee WWR, Ling KL, et al. Consensus and contentious statements on the use of probiotics in clinical practice: a South East Asian Gastro-Neuro Motility Association Working Team Report. J Gastroenterol Hepatol 2018; 33: 1707-16.

43. Merenstein D, Salminen S, Team W.G.O.R. World Gastroenterology Organisation Global Guidelines - probiotics and prebiotics. World Gastroenterology Organisation 2017.

44. Song KH, Jung HK, Kim HJ, et al. Clinical Practice Guidelines for irritable bowel syndrome in Korea, 2017 Revised Edition. J Neurogastroenterol Motil 2018; 24: 197-215.

45. Pietrzak A, Skrzydło-Radomańska B, Mulak A, et al. Guidelines on the management of irritable bowel syndrome. Gastroenterology Rev 2018; 13: 259-88.

46. Ford AC, Moayyedi P, Chey WD, et al. American College of Gastroenterology Monograph on management of irritable bowel syndrome. Am J Gastroenterol 2018; 113 (Suppl 2): 1-18.

47. Lacy BE, Pimentel M, Brenner DM, et al. ACG Clinical Guideline: Management of Irritable Bowel Syndrome. Am J Gastroenterol 2021; 116: 17-44.

48. Dumitrascu DL, Baban A, Bancila I, et al. Romanian guidelines for nonpharmacological therapy of IBS. J Gastrointest Liver Dis 2021; 30: 291-306

49. McKenzie YA, Alder A, Anderson W, et al. British Dietetic Association evidence-based guidelines for the dietary management of irritable bowel syndrome in adults. J Human Nutrition Dietetics 2012; 25: 260-74.

Received: 23.08.2021

Accepted: 30.09 .2021 